\title{
EFECTOS DE LA MÚSICA-DANZA Y DEL REFUERZO POSITIVO EN LA CONDUCTA DE PERSONAS CON DISCAPACIDAD MÚLTIPLE
}

\author{
Daisy María Mora Zúñiga, Walter Salazar y Rocío Valverde \\ Escuela de Educación Física y Deportes \\ Universidad de Costa Rica, San José, Costa Rica \\ e-maildmmora@cariari.ucr.ac.cr
}

\begin{abstract}
Resumen
Mora Zúñiga, D. M., Salazar, W., y Valverde, R. (2001). Efectos de la música-danza y del refuerzo positivo en la conducta de personas con discapacidad múltiple. Revista de Ciencias del Ejercicio y la Salud, 1(1), 19-33. El propósito de este estudio fue determinar los efectos agudo y crónico de: la música-danza y del refuerzo positivo en conductas no deseadas y el seguimiento de órdenes en una población con discapacidad múltiple. Los participantes, de ambos sexos $(n=13$, edad $\boldsymbol{X}=23.38 \pm 7.87$ años), fueron divididos aleatoriamente en tres grupos: música-danza $(\boldsymbol{M D}, n=4)$, refuerzo positivo $(\boldsymbol{R P}, n=5)$ y grupo control $(\boldsymbol{G C}, n=4)$. Las variables medidas fueron conductas no deseadas $(\boldsymbol{C N D})$ y seguimiento de órdenes $(\mathbf{S O})$. Los grupos $\boldsymbol{M D}$ y $\boldsymbol{R P}$ recibieron cuatro sesiones por semana, de veinte minutos cada una, durante tres semanas. Para determinar el efecto crónico se realizó un registro de línea base $(\boldsymbol{L B})$ de veinte minutos durante cuatro días (pretest) para $\boldsymbol{C N D}$ y un registro de línea base $(\boldsymbol{L B})$ de cuarenta órdenes durante cuatro días (pretest) para SO. Luego de la última semana de tratamiento se aplicó el postest 1, y quince días después se realizó el postest 2, bajo las mismas condiciones del pretest, tanto para CND como SO. Las mediciones para determinar el efecto agudo se hicieron durante una sesión, para CND y SO. El análisis estadístico consistió en ANOVAS de dos vías (3 grupos $x 3$ mediciones) tanto para el efecto agudo como para el crónico. Las CND disminuyeron significativamente $(p<0.05)$ de forma aguda con ambos métodos, siendo más efectivo $\boldsymbol{M D}$ que $\boldsymbol{R P}(p<0.05)$; sin embargo, crónicamente solo disminuyeron con $\boldsymbol{R P}(p<0.05)$, pero no hubo efecto de retención ( $p>0.05)$. SO fue significativamente mejor $(p<0.05)$ con $\mathbf{M D}$ tanto crónica como agudamente. EL GC no mostró cambios en ninguna medición. Futuros estudios deberían investigar los efectos de MD y $\boldsymbol{R P}$ en otras variables, como: comportamiento no verbal o movimientos voluntarios, en una población con discapacidad múltiple. PALABRAS CLAVES: música, danza, procesos psicológicos, discapacidad.
\end{abstract}

\section{INTRODUCCION}

La conducta humana es un tema muy controversial $y$ ha sido ampliamente discutido por diferentes campos de estudio del hombre: psicología (Skinner, 1975), etología (Díaz, 1994; Eibi-Eibesfeldt, 1974; Hall, 1989; Lorenz, 1984), pedagogía (Piaget, 1989 y 1994), psiquiatría (Cabanas, 1979).

La conducta se puede definir como lo que hace un individuo bajo condiciones específicas. (Sulzer-Azaroff y Mayer, 1998) y se aprende en función de las consecuencias (Cabezas y Fonseca, 1992). Un comportamiento tiene siempre una causa que se puede hallar tanto en estímulos sensoriales externos como internos (Eibi-Eibesfeldt, 1974; Ricci y Cortesi, 1977).

Así, parte de la personalidad está determinada por la interioridad como ser individual, y otra parte, por la interacción con el medio físico y por las relaciones familiares y sociales. Por ello las conductas deben ser positivas, adecuadas y funcionales de acuerdo con el medio social en que se desenvuelve. Basándose en esto, las conductas positivas, adecuadas y funcionales, benefician las relaciones familiares, sociales, escolares, y en general 
facilitan la adquisición de elementos positivos que le ayudan al individuo a desenvolverse "debidamente" y adquirir nuevos conocimientos (Bukley y Walker, 1970; Pelechano, 1975; Sulzer y Mayer, 1988).

Por el contrario, todas aquellas conductas consideradas como "inadecuadas y no funcionales", o conductas no deseadas, perjudican el "desarrollo adecuado" del ser humano, interfiriendo en la adquisición de otras conductas beneficiosas y que faciliten los aspectos cognitivos. Sin embargo, las conductas inadecuadas y no funcionales ocurren de vez en cuando en el repertorio conductual de todo individuo (Paniagua 1994).

En las personas con discapacidad, las conductas inadecuadas y no funcionales se presentan con mayor frecuencia e intensidad, lo que limita todas sus capacidades cognoscitivas y de adquisición de conductas que faciliten el aprendizaje de nuevas destrezas y actitudes para su desarrollo integral como ser humano (Borden y otros, 1970).

No obstante, la conducta puede modificarse, eliminando o disminuyendo las conductas, y por consiguiente, aumentando o iniciando las conductas beneficiosas y funcionales (Blockhan y Silberman, 1973).

La modificación de conducta puede realizarse desde diferentes metodologías. Las técnicas más utilizadas para debilitar conductas inadecuadas y fortalecer conductas deseadas son las técnicas de reforzamiento. Principalmente se usa el refuerzo positivo, que se da cuando una respuesta es seguida por una consecuencia positiva o agradable, entonces aumenta la posibilidad de que se repita en lo futuro, es decir, la respuesta se fortalece (Granell, 1979; Ribes, 1975; Walker y Shea, 1987). Esto se basa en la teoría de estímulo-respuesta-consecuencia (Franks, 1991; Skinner,1975).

Esta técnica ha sido utilizada durante muchos años, por psicólogos, educadores y padres de familia, obteniendo mejoras significativas en diferentes aspectos de la conducta (por ejemplo: Beirute, 1974; Broden y otros, 1970; Cabezas, 1989; Carvajal, 1989; Ferster, 1961; Long y otros; 1999; Mora, 1982; Piazza y otros, 2000). En educación física, el $97 \%$ de las técnicas de manejo conductual usadas por el profesor están basadas en el condicionamiento operante (Siedentop y Rushall, 1972). Igualmente, los educadores físicos que trabajan con personas con retardo mental profundo, normalmente utilizan técnicas del refuerzo operante, para el manejo conductual (Culver, 1989; Stranathan y otros, 1997).

Para lograr sus objetivos, habitualmente se usan diferentes tipos de reforzadores, principalmente los sociales y los comestibles.

Otros autores, como Silliman y otros (1998), utilizan la música como reforzador positivo trabajando con niños de diez años ciegos y con retardo mental profundo, para determinar la influencia de la música en el mejoramiento de destrezas de motora gruesa y la transferencia de éstas a un nuevo ambiente. El tratamiento se aplicó tres veces por día, durante diez días, 30-40 minutos cada sesión. Dos semanas después de finalizar el tratamiento generalizaron las destrezas (mejoraron y transfirieron), pero esto decreció a los tres meses de haber concluido. En este caso específico, la música constituye un elemento reforzador. usar

En otros estudios, se emplea la música como el estímulo, y normalmente va acompañada de la danza, ya que la música y la danza constituyen un elemento importante en la vida de las personas, sin importar la edad, el sexo, la clase social, los aspectos culturales. No obstante, Crain y otros (1984), en una revisión de literatura encontraron que existe una polémica en cuanto a la importancia de la danza y los beneficios físicos, sociales y psicológicos que pueden esperar las personas con retardo mental al ser expuestos a un programa de danza terapia (Calder, 1972; Kraus, 1978; Moran y Calakian, 1974).

Otros autores, de igual forma han encontrado que los programas de música y/o danza no contribuyen al mejoramiento de 
aspectos que intervienen en el aprendizaje, en la autoestima y en la socialización de personas con necesidades especiales que participan en ellos (Kavaler, 1974; Ozols y Revuelta,1996).

A pesar de esto, Brooks (1989) piensa que los ejercicios de danza-movimiento traen varios beneficios a las personas con problemas de aprendizaje, tales como:

-mejoramiento de la autoimagen,

- desarrollo de la sensación kinestésica, que ayuda en la movilidad, lateralidad y direccionalidad,

-incremento en la motivación,

-desarrollo de la conexión mente-cuerpo,

-desarrollo de la cooperación social y trabajo grupal.

Otros autores han aplicado programas de música y/o danza en esta misma población encontrando que éstos sí benefician el aprendizaje, la autoestima y la socialización (Berrol, 1978; Berrol, 1981; Boettinger, 1978; Dorney y otros, 1992; Freundlich y otros, 1989; Jatib, 1998; Ohwaki, 1976; Riordan, 1989; Weltman 1986; Wise, 1981).

Neddo (1986), estudió el efecto de un programa de danza aeróbica en el perfil de riesgo en mujeres universitarias con patrones de conducta tipo A, extrovertidas, siempre tienen que estar en movimiento, con problemas de salud debido al estrés. Las asignó a dos grupos: experimental con el programa de danza aeróbica y control. Después de doce semanas, el grupo experimental disminuyó significativamente los patrones de conducta tipo A.

Masako y Chiyo (1996), trabajaron con dos grupos para determinar el efecto de una pieza de música y el estímulo de danza en la imagen psicológica; al grupo experimental se le midió la respuesta a una pieza musical, bajo el estímulo de la danza; al grupo control se le midió la respuesta a la pieza musical, pero sin el estímulo de la danza. Encontraron mayores mejoras en el grupo experimental.

En cuanto a estudios con población especial, se encuentran pocos que relacionan la música y/o la danza con la conducta.
Jay (1991) realizó un estudio con niños preescolares con discapacidad, investigó el efecto de un programa de danza en la imaginación, la influencia y la originalidad. Un grupo trabajó con un programa de danza, basado en experiencias sensoriales, utilizando las acciones de esfuerzo de Laban, el otro grupo recibió educación física adaptada sin danza. Encontró que con el programa de danza mejoraron significativamente la imaginación, pero no la influencia y la originalidad.

Crain y otros (1984), trabajaron con un grupo de trece estudiantes adolescentes, de 13 a 15 años de edad, siete hombres y seis mujeres, con retardo mental. El programa consistió en 30 sesiones de actividades de danza, de 30 minutos cada sesión, durante 10 semanas, aplicado tres veces por semana. El programa fue dividido en cinco unidades: 1movimientos de orientación, 2- movimientos de exploración, 3- fundamentos de danza, 4ritmo y 5- danza. Al finalizar el tratamiento cuatro estudiantes mejoraron conductas sociales y físicas, siete mejoraron solo las conductas sociales o las físicas y los otros dos no mostraron cambios significativos. No se encontró efecto de transferencia, además del hecho de que algunas conductas estudiadas se vieron afectadas por factores externos.

Por estas razones y muchas otras es que se ha utilizado la música y la danza con fines terapéuticos. De aquí nacen algunos conceptos importantes:

Músico Terapia. Loroño y del Campo (1987), definen la músico terapia como el "campo apasionante y complejo de la terapia y la reeducación por medio de la aplicación de la música en su sentido más amplio".

Más recientemente Alvin (1990), la define como el "uso dosificado de la música en el tratamiento, la rehabilitación, la educación y el adiestramiento de adultos y niños que padecen trastornos físico, mentales o emocionales". En 1998, Freundlich y otros, plantean que "la músico terapia involucra el uso de la música como influencia positiva en 
cambios conductuales y ayuda a cada individuo a incrementar su potencial“".

Danza Terapia. Frieder y Eckstein (1981), definen la danza terapia como el "uso psicoterapéutico del movimiento como proceso que busca la integración emocional de la persona". Más recientemente la American Dance Therapy Association (1999), define la danza terapia como "el uso psicoterapéutico del movimiento como un proceso que fomenta la integración emocional, cognitiva, social y física del individuo".

Según Levy (1988), "la premisa básica de la danza terapia es que el movimiento del cuerpo refleja estados internos emocionales y que el cambio en la forma de moverse, origina cambios psicológicos, de esta forma el movimiento se concibe como un comunicador primario de los estados profundos emocionales y mentales del ser humano". Por lo tanto, según Schmais (1974) la danza terapia: envuelve la comunicación a través de los movimientos del cuerpo y está basada sobre tres postulados: 1- Reflejos de movimientos de rasgos personales $y$ habilidades, 2- Relación entre el terapeuta y el apoyo al cliente, 3- El cambio conductual a través del movimiento.

En las personas con discapacidad múltiple también es indispensable mejorar su salud mental y permitir la expresión de su interioridad utilizando el trabajo con música y con danza. Y con ello se pueden estimular otras condiciones y actitudes que faciliten sus interrelaciones sociales, familiares, escolares. De aquí surge la necesidad de utilizar música-danza para trabajar con personas con discapacidad múltiple:

Música-Danza. "Manifestación libre e inducida de la danza-movimiento, que genere cada persona al escuchar la música".

Viendo la importancia de la manifestación del movimiento y el poder terapéutico de la música y la danza y su posible contribución al cambio conductual; por otro lado, sabiendo los efectos beneficiosos del refuerzo positivo en la modificación de conducta; surge la inquietud de determinar qué método: refuerzo positivo (tradicional) o música-danza (propuesto) es más efectivo en la disminución de presentación de conductas no deseadas y en el incremento en la respuesta positiva al seguimiento de órdenes en personas con discapacidad múltiple.

\section{METODOLOGÍA}

\section{Participantes}

Se contó con una muestra de trece participantes (ocho mujeres y cinco hombres), internos del Hogar Luz, Tres Ríos. Con edades comprendidas entre los 13 y 31 años (edad X = 23.38+7.87años) los cuales presentan discapacidad múltiple en grado severo, lo que incluye retardo mental, parálisis cerebral, autismo, trastornos emocionales, problemas de lenguaje, problemas defectivos visuales.

\section{Instrumentos de medición}

Se pasaron los siguientes instrumentos de medición:

-Tablas de cotejo de duración, para las diferentes conductas no deseadas:

$\rightarrow$ balanceo de tren superior,

$\rightarrow$ movimiento de manos,

$\rightarrow$ balanceo de cabeza,

$\rightarrow$ berrinche,

$\rightarrow$ contacto físico con otra persona $u$ objeto.

-Tablas de cotejo de frecuencia, para el seguimiento de órdenes:

$$
\begin{array}{ll}
\rightarrow \text { venga, } & \rightarrow \text { vaya }, \\
\rightarrow \text { tome } & \rightarrow \text { deme }, \\
\rightarrow \text { suba }, & \rightarrow \text { baje }, \\
\rightarrow \text { lance }, & \rightarrow \text { apañe }, \\
\rightarrow \text { siéntese, } & \rightarrow \text { acuéstese. }
\end{array}
$$

\section{Procedimientos de medición}

Se determinaron y operacionalizaron las conductas no deseadas de cada sujeto para ser modificadas. Se establecieron diez 
órdenes diferentes, de acciones escasas en su repertorio conductual, que fueran de importancia para sus actividades diarias y que constituyeran una base importante para el aprendizaje de nuevas destrezas y habilidades.

Los sujetos se dividieron aleatoriamente en tres grupos de acuerdo con el tratamiento por recibir. Cuatro personas en el tratamiento de música y danza $(\boldsymbol{M D})$, cinco personas en el tratamiento de refuerzo positivo $(\boldsymbol{R P})$ y cuatro personas como grupo control $(\boldsymbol{G C})$.

Los tratamientos se realizaron cuatro sesiones por semana, de veinte minutos cada una, durante tres semanas. Fueron aplicados en un aula asignada para dicho fin, fuera de su espacio de estar diario. Se realizaron de la siguiente manera:

-Refuerzo Positivo $(\boldsymbol{R P})$ :

-se trabajó de forma individual,

-durante cada sesión y para cada conducta:

$\rightarrow$ Se modelaba la conducta deseada,

$\rightarrow$ se pedía al sujeto que realizara la conducta,

$\rightarrow$ se premiaba, de forma inmediata, cada vez que la ejecutaba correctamente,

$\rightarrow$ se inhibía la conducta no deseada cada vez que se presentaba, utilizando la orden "no" y con intervención directa (contacto físico).

-desde el inicio del tratamiento se utilizaron solamente reforzadores sociales (muy bien, excelente, qué bueno, eso, qué bien).

-Música-Danza (MD):

-se trabajó de forma grupal,

-la danza fue estimulada, pero se respetó la respuesta que cada individuo generó y se siguió trabajando sobre esa línea, la música marcó la pauta del tipo de movimiento realizado,

-se utilizó Reggae (Bob Marley) y Gipsy Kings,

-si surgían conductas no deseadas, no eran inhibidas.

-Grupo Control $(\boldsymbol{G C})$ : -fueron trasladados todos juntos hacia la sala donde se realizaban los otros tratamientos,

-no se realizó ningún tipo de intervención (excepto en el caso de la autoagresión),

-normalmente permanecieron sentados, acostados o caminando por toda la sala.

Para determinar el efecto crónico a todos los grupos se les aplicó el pretest, mediante un registro de línea base $(\boldsymbol{L B})$ de las conductas no deseadas, durante cuatro días, veinte minutos cada día; y un registro de $\boldsymbol{L} \boldsymbol{B}$ de seguimiento de órdenes, durante cuatro días, cuarenta órdenes cada día.

Se aplicaron los tratamientos respectivos.

Al finalizar el tratamiento se volvió a criterios de $\boldsymbol{L} \boldsymbol{B}$, y se aplicó el postest 1 para las conductas no deseadas, durante cuatro días veinte minutos cada día; y para el seguimiento de órdenes, durante cuatro días, cuarenta órdenes cada día.

Quince días después del postest 1, se aplicó el postest 2 bajo las mismas condiciones del pretest y el postest 1 , tanto para las conductas no deseadas como para el seguimiento de órdenes.

El efecto agudo se midió durante una sesión diferente para cada variable, comparando mediciones realizadas antes, durante y después la sesión. Para las conductas no deseadas, se aplicó el postest veinte minutos antes, el test durante y el postest veinte minutos después de la sesión número seis. Para el seguimiento de órdenes, se registró el seguimiento de cuarenta órdenes antes (pretest), cuarenta órdenes durante (test) y cuarenta órdenes después de la sesión (postest), durante la sesión número siete.

\section{Análisis estadístico}

La estadística descriptiva consistió en promedios y desviaciones estándar de los grupos y de las pruebas.

En la estadística inferencial, para determinar el efecto crónico, tanto para la 
variable de conductas no deseadas como para la variable de seguimiento de órdenes se aplicaron ANOVAS de dos vías (3 grupos $\mathrm{x}$ 3 pruebas). En ambos casos, conductas no deseadas y seguimiento de órdenes, se aplicó cada test durante cuatro días para una medición más estable, pero estos valores fueron promediados para el análisis estadístico.

Para comprobar el efecto agudo en ambas variables, se aplicaron ANOVAS de dos vías (3 grupos x 3 pruebas).

A las interacciones significativas se les dio un seguimiento de un análisis de los efectos simples (Keppel, 1982, pág. 312). Y los efectos simples que dieron significativos se siguieron con un post-hoc de Tukey (Keppel, 1982, pág. 155).

Se obtuvo el tamaño del efecto (ES) (Thomas, Salazar y Landers, 1991) para comparar los dos tratamientos y el grupo control en las dos variables medidas ( $C N D$ y $\boldsymbol{S} \boldsymbol{O})$, tanto en el efecto agudo como en el efecto crónico.

\section{RESULTADOS}

A continuación se presentan los resultados de la estadística descriptiva, promedio y desviación estándar de los grupos, en las tres mediciones hechas en cada variable.

En el cuadro $\mathrm{N}^{\circ} 1$ se pueden apreciar los promedios y la desviación estándar de los datos obtenidos en las pruebas aplicadas durante el efecto agudo (sesión número seis) y el efecto crónico de las conductas no deseadas en los diferentes grupos.

Cuadro $\mathrm{N}^{\mathbf{0}} \mathbf{1}$

Efecto Agudo y Efecto Crónico Conductas no Deseadas Medidas en 1200 Segundos Música y Danza (MD), Refuerzo Positivo (RP) y Grupo Control (GC)

\begin{tabular}{cccc}
\hline Efecto agudo & Pretest (s) & Test (s) & Postest (s) \\
\hline MD & $1076.00 \pm 137.45$ & $276.25 \pm 48.38$ & $321.75 \pm 43.29$ \\
RP & $998.40 \pm 166.62$ & $538.40 \pm 65.94$ & $572.40 \pm 79.38$ \\
GC & $937.75 \pm 184.80$ & $939.75 \pm 204.85$ & $915.75 \pm 207.55$ \\
\hline Efecto crónico & Pretest (s) & Postest 1 (s) & Postest 2 (s) \\
\hline MD & $1017.94 \pm 125.10$ & $863.38 \pm 45.48$ & $1053.1 \pm 33.18$ \\
RP & $941.55 \pm 152.13$ & $567.35 \pm 53.91$ & $971.70 \pm 68.11$ \\
GC & $919.56 \pm 170.41$ & $940.88 \pm 199.25$ & $943.31 \pm 198.31$ \\
\hline \multicolumn{4}{r}{$*$ los datos indican el promedio y la desviación estándar }
\end{tabular}

En el cuadro $\mathrm{N}^{\circ} 2$ se detallan los promedios y la desviación estándar de los datos obtenidos en las pruebas aplicadas durante el efecto agudo y el efecto crónico del seguimiento de órdenes en los diferentes grupos.

Seguidamente se presentan los resultados de la estadística inferencial.
Para determinar el efecto agudo de las conductas no deseadas se realizó una ANOVA de dos vías (3 grupos $\mathrm{x} \quad 3$ mediciones) donde resultó significativa la interacción, $\mathrm{F}(4,20)=19.62, p<0.05, \varpi^{2}=$ $21.35 \%$. 


\section{Cuadro $\mathrm{N}^{\circ} 2$ \\ Efecto Agudo y Efecto Crónico Seguimiento de Ordenes Basándose en un Máximo de 40 Ordenes Música y Danza (MD), Refuerzo Positivo (RP) y Grupo Control (GC)}

\begin{tabular}{cccc}
\hline Efecto agudo & Pretest & Test & Postest \\
\hline MD & $17.83 \pm 11.20$ & $32.67 \pm 8.59$ & $30.80 \pm 12.28$ \\
RP & $16.53 \pm 6.67$ & $22.67 \pm 10.45$ & $16.13 \pm 5.88$ \\
GC & $18.33 \pm 6.06$ & $19.17 \pm 7.73$ & $19.33 \pm 5.13$ \\
\hline Efecto crónico & Pretest & Postest 1 & Postest 2 \\
\hline MD & $17.21 \pm 4.10$ & $34.33 \pm 8.32$ & $20.42 \pm 11.35$ \\
RP & $14.90 \pm 5.91$ & $21.23 \pm 9.72$ & $16.23 \pm 4.69$ \\
GC & $16.96 \pm 5.10$ & $17.37 \pm 7.34$ & $18.92 \pm 4.11$ \\
\hline
\end{tabular}

* los datos indican el promedio y la desviación estándar

A la interacción se le dio seguimiento con el análisis de los efectos simples y se vio que había diferencia significativa entre grupos,

\section{Gráfico No1}

Interacción entre los Promedios Efecto Agudo Conductas no Deseadas

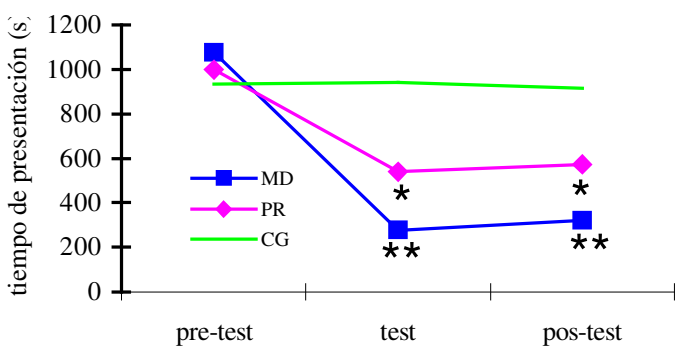

*: estadísticamente diferente de los que no tienen * **: estadísticamente diferente de los demás promedios

Para darle seguimiento se realizó el posthoc de Tukey, tanto para los grupos como para las mediciones, donde se demostró que había diferencia significativa entre $\boldsymbol{R P}$ y $\boldsymbol{G C}$ en el test y el postest; entre $\boldsymbol{M D}$ y los otros dos, $\boldsymbol{R P}$ y $\boldsymbol{G C}$, en el test y el postest; en $\boldsymbol{M D}$ entre el pretest, y los otros, test y postest; y en $\boldsymbol{R P}$ entre el test y los otros, test y postest.
MD, $\mathrm{F}(2,20)=71.86, p<0.05$ y $\boldsymbol{R P}, \mathrm{F}(2,20)$ $=36.46, p<0.05$.

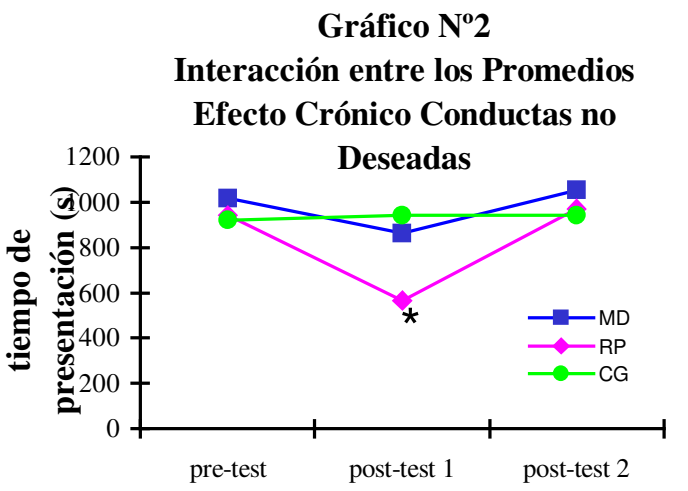

*: estadísticamente diferente de los otros promedios

Esto significa que ambos tratamientos fueron efectivos con respecto al grupo control y al pretest, sin embargo fue significativamente mayor el efecto agudo de música-danza, con respecto al refuerzo positivo, tanto durante como al finalizar la sesión. Se detalla en el gráfico $\mathrm{N}^{\mathrm{o}} 1$. 
Gráfico No3

Interacción entre los Promedios

Efecto Agudo Seguimiento de

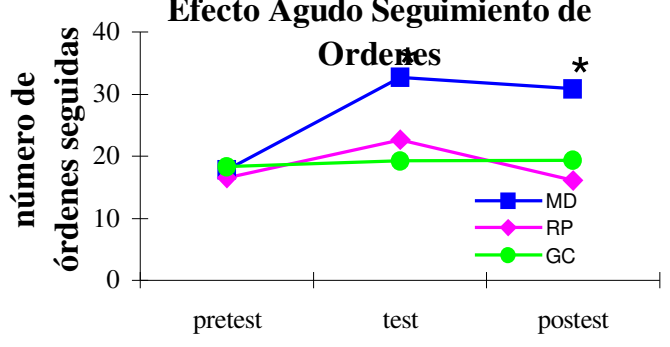

*: estadísticamente diferente de los otros promedios

Para encontrar el efecto crónico de las conductas no deseadas se realizó una ANOVA de dos vías (3 grupos $\mathrm{x} 3$ mediciones) donde resultó significativa la interacción, $\mathrm{F}(4,20)=10.74, p<0.05, \varpi^{2}=$ $17.77 \%$.

A la interacción se le dio seguimiento con el análisis de los efectos simples y se vio diferencia significativa entre grupos $\boldsymbol{M D}$,

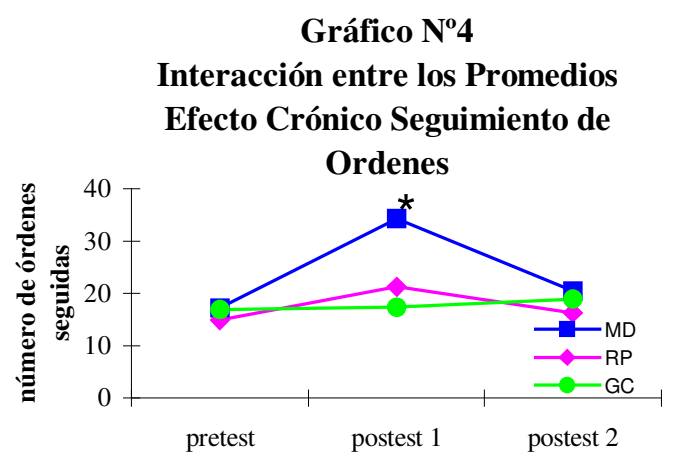

*: estadísticamente diferente de los otros promedios

$\mathrm{F}(2,20)=9.76, p<0.05$ y $\boldsymbol{R P}, \mathrm{F}(2,20)=$ 4.71, $p<0.05$.

Para darle seguimiento se realizó el posthoc de Tukey, donde se demostró que había diferencia significativa entre $\boldsymbol{R P}$ y los otros, $\boldsymbol{M D}$ y $\boldsymbol{G} \boldsymbol{C}$ en el postest 1; y en $\boldsymbol{R P}$ entre el postest 1 y los otros, pretest y postest 2 .

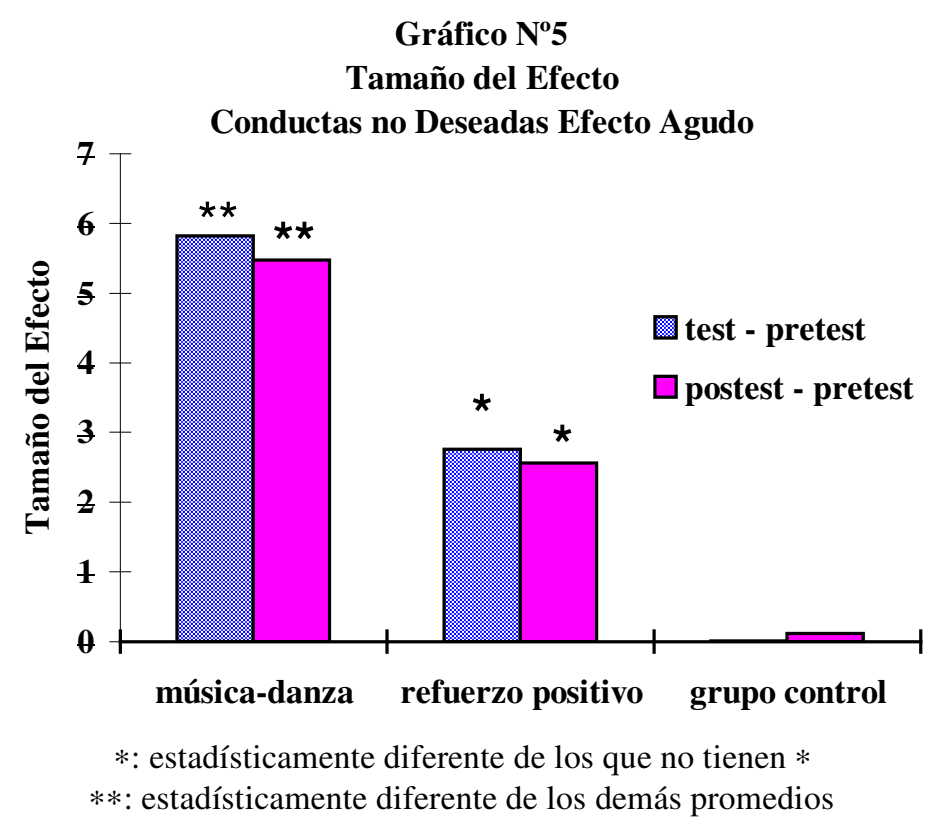

Lo que significa que crónicamente solo fue efectivo el refuerzo positivo, pero no se da un efecto de retención. Esto se ve en el gráfico $\mathrm{N}^{\mathrm{o}} 2$. 
Para encontrar el efecto agudo del seguimiento de órdenes se realizó una ANOVA de dos vías (3 grupos $\mathrm{x} 3$ mediciones) donde resultó significativa la interacción, $\mathrm{F}(4,20)=7.63, p<0.05, \varpi^{2}=$ $9.86 \%$.
A la interacción se le dio seguimiento con el análisis de los efectos simples y se vio que había diferencia significativa entre grupos $\boldsymbol{M D}, \mathrm{F}(2,20)=32.97, p<0.05$ y $\boldsymbol{R} \boldsymbol{P}$, $\mathrm{F}(2,20)=9.68, p<0.05$.

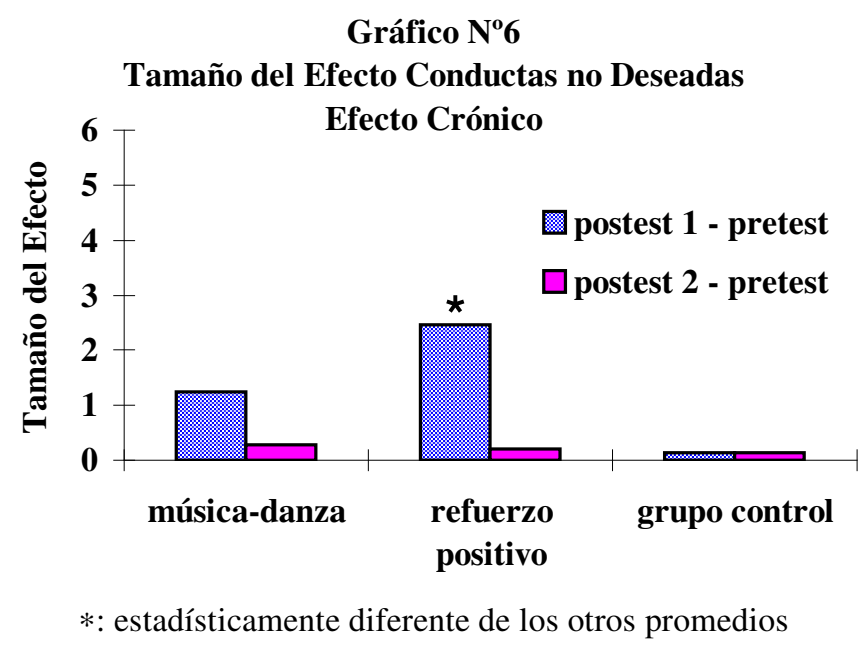

Para darle seguimiento se realizó el posthoc de Tukey, tanto para los grupos como para las mediciones, donde se demostró que había diferencia significativa entre $\boldsymbol{M D}$ y los otros dos, $\boldsymbol{R P}$ y $\boldsymbol{G C}$ en el test y el postest; y en $\boldsymbol{M D}$ entre el pretest y las otras dos mediciones, test y postest.

Esto significa que solamente fue efectivo el tratamiento propuesto, música danza, tanto

durante, como al finalizar la sesión. Esto se muestra en el gráfico $\mathrm{N}^{\circ} 3$.

Para encontrar el efecto crónico del seguimiento de órdenes se realizó una ANOVA de dos vías (3 grupos $\mathrm{x} \quad 3$ mediciones) donde resultó significativa la interacción, $\mathrm{F}(4,20)=12.77, p<0.05, \varpi^{2}=$ $11.75 \%$.

Gráfico $\mathbf{N}^{0} 7$

Tamaño del Efecto Seguimiento de Ordenes

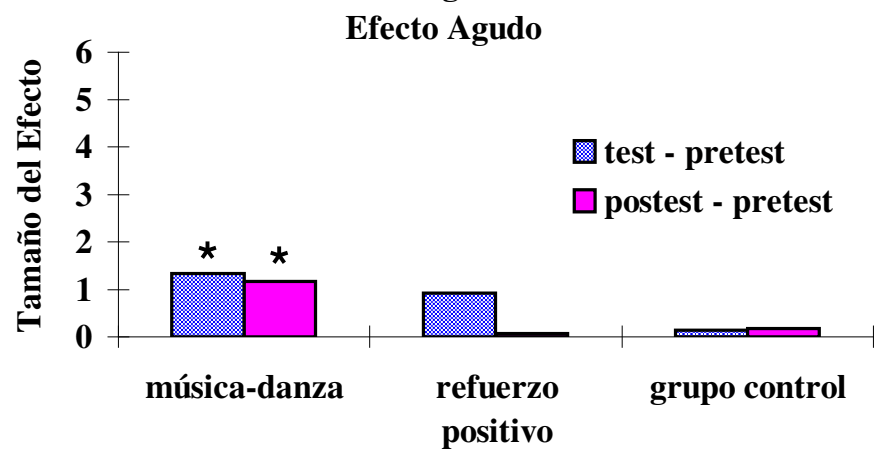

*: estadísticamente diferente de los otros promedios 
A la interacción se le dio seguimiento con el análisis de los efectos simples y se vio que había diferencia significativa entre grupos $\boldsymbol{M D}, \mathrm{F}(2,20)=48.65, p<0.05$ y $\boldsymbol{R} \boldsymbol{P}$, $\mathrm{F}(2,20)=10.26, p<0.05$.

Para darle seguimiento se realizó el posthoc de Tukey, tanto para los grupos como para las mediciones, donde se demostró que había diferencia significativa entre $\boldsymbol{M D}$ y los otros, $\boldsymbol{R P}$ y $\boldsymbol{G C}$ en el postest 1; y en $\boldsymbol{M D}$ entre el postest 1 y las otras dos mediciones, pretest y postest 2 .

Al igual que en el efecto agudo, se observa una mejora significativa solamente con música-danza, comparado con el refuerzo positivo y el grupo control, sin embargo no hay efecto de retención. Esto se muestra en el gráfico $\mathrm{N}^{\circ} 4$.

A continuación se presentan cuatro gráficos resumen, donde se compara el tamaño del efecto del pretest, test y postest para las dos variables $(\boldsymbol{C N D}$ y $\boldsymbol{S O})$, medidas de forma aguda, en los tres tratamientos $(\boldsymbol{M D}, \boldsymbol{R P}$ y $\boldsymbol{G C})$, y de los dos postest con respecto al pretest, para las dos variables $(\boldsymbol{C N D}$ y $\boldsymbol{S O})$, medidas de forma crónica, en los tres tratamientos (MD, $\boldsymbol{R P}$ y $\boldsymbol{G C})$.

En el gráfico $\mathrm{N}^{\circ} 5$ se presenta el tamaño del efecto en conductas no deseadas del postest 1 y del postest 2 con respecto al pretest, medido en el efecto agudo. En el cual se observa una superioridad de la música- danza con respecto al refuerzo positivo, a pesar de que este último también fue efectivo, relacionado con su pretest y el grupo control.

En el gráfico $\mathrm{N}^{\circ} 6$ se presenta el tamaño del efecto en conductas no deseadas del postest $1 \mathrm{y}$ del postest 2 con respecto al pretest, determinado en el efecto crónico. Con esto se observa una mejora significativa del refuerzo positivo con respecto al grupo control y a música-danza.

En el gráfico $\mathrm{N}^{\circ} 7$ se presenta el tamaño del efecto para el seguimiento de órdenes, medido en el efecto agudo, del test y del postest con respecto al pretest. $\mathrm{Y}$ en el gráfico $\mathrm{N}^{\circ} 8$ se presenta el tamaño del efecto para el seguimiento de órdenes, medido en el efecto crónico, del postest 1 y del postest 2 con respecto al pretest. En ambos se corrobora la eficacia de música-danza con respecto al grupo control y al refuerzo positivo, pese a que en este último, los tamaños del efecto, también son altos.

En resumen, se denota una clara superioridad del método propuesto, músicadanza, sobre el método tradicional, refuerzo positivo en todos los casos, excepto en el efecto crónico de las conductas no deseadas; pese a que también el refuerzo positivo fue efectivo en el efecto agudo de las conductas no deseadas, música-danza fue superior.

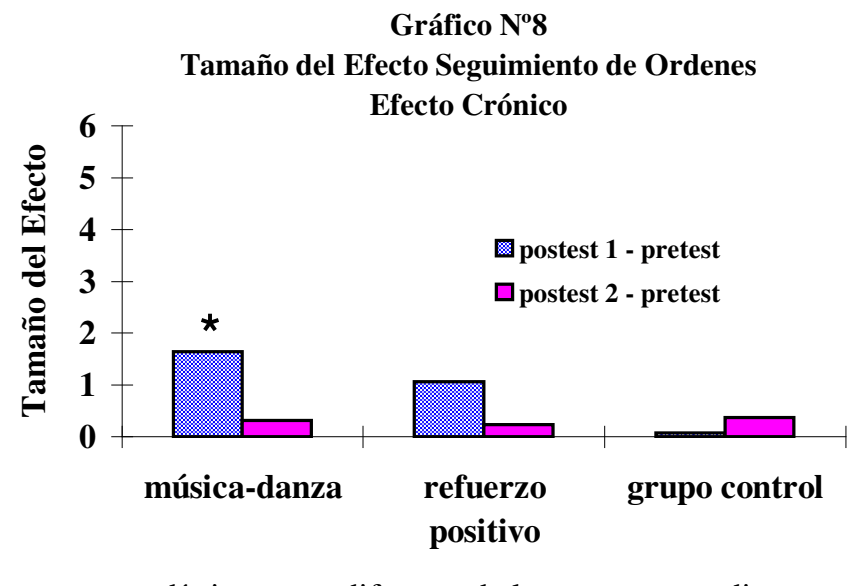

*: estadísticamente diferente de los otros promedios 


\section{DISCUSIÓN}

La tendencia conductista ha sido aceptada por profesionales de diferentes áreas (psicología, educación regular, educación especial, etc.), quienes han utilizado el refuerzo positivo con fines de modificación de conducta obteniendo resultados favorables (Beirute, 1974; Broden y otros, 1970; Cabezas, 1989; Carvajal, 1989; Ferster, 1961; Long y otros, 1999; Mora, 1982; Richman y otros, 1999; Russell $\mathrm{y}$ otros, 1999).

Por otro lado, la música y la danza se ha implementado con fines terapéuticos en muchos y muy variados campos, dentro de los cuales se encuentra el manejo o cambio conductual en población especial (Berrol, 1978; Berrol, 1981; Boettinger, 1978; Crain y otros, 1984; Dorney y otros, 1992; Freundlich y otros, 1989; Jatib, 1998; Jay (1991); Mora y Salazar, 1999; Ohwaki, 1976; Riordan, 1989; Weltman 1986; Wise, 1981).

En este estudio se comparan ambos métodos, tanto aguda como crónicamente: refuerzo positivo (método tradicional) y música-danza (método propuesto), lo que implica algo novedoso e importante con respecto al manejo conductual con personas con discapacidad múltiple,

Los resultados indican que el método nuevo, propuesto para el estudio, músicadanza, en términos generales (aunque no en todas la variables) fue más efectivo que el método utilizado tradicionalmente para el manejo conductual, refuerzo positivo.

En cuanto a la disminución de conductas no deseadas, ambos métodos son efectivos de forma aguda con respecto al grupo control, pero es importante hacer notar que a pesar de ello, la música-danza fue significativamente superior que el refuerzo positivo.

Por lo tanto, en ambos casos disminuye la duración de presentación aguda de conductas no deseadas. En el grupo de $\boldsymbol{R} \boldsymbol{P}$, durante la sesión siempre que se iniciaba la presentación de alguna conducta no deseada, era inhibida inmediatamente por el investigador, (como la técnica lo sugiere), lo que contribuye a su disminución, además de reforzar socialmente cuando realiza las conductas deseadas, quizás esto contribuyó a que se mantuvieran disminuidas al finalizar la sesión. En el grupo $\boldsymbol{M D}$, durante la sesión, las conductas no se presentaban tan frecuentemente como antes de la misma; se podría decir, que momentáneamente se olvidaban de las conductas para moverse, para danzar, quizás debido a la importancia de la música para ellos, que constituye un estímulo agradable, que favorece la danza y la disposición para realizar otras actividades "importantes", dejando de lado las CND, manteniendo ésta condición al finalizar la sesión. Esto se respalda con el tamaño del efecto determinado.

La música-danza es un método de fácil aplicación, y prácticamente ellos danzan de forma inmediata al escuchar la música de su agrado. Esto se sustenta en los resultados encontrados previamente por Mora y Salazar (1999), ya que estudiando la misma población determinaron que la música y la danza disminuyen significativamente, de forma aguda, la frecuencia y/o el tiempo de presentación de conductas no deseadas, y más importante, se encontró que la combinación de ambas música-danza (método propuesto también en este estudio) fue indiscutiblemente superior que cada uno por separado.

En cuanto al efecto crónico sucedió una situación un tanto particular, pues a pesar de que música-danza fue más efectivo de forma aguda, crónicamente, el grupo que trabajó con el refuerzo positivo, fue significativamente superior a los otros dos grupos. Esta es una técnica establecida que ha sido utilizada durante muchos años y ha dado resultados favorables en cuanto a modificación conductual, lo que respalda estos resultados. esto se ejemplifica con algunas de las investigaciones realizadas en esta área, descritas a continuación:

Esto apoya los resultados obtenidos por Beirute (1974), quien, con la ayuda de los 
maestros de escuela, aplicó un programa con el uso de refuerzo positivo para modificar la conducta de niños con retardo mental. Al finalizar el tratamiento, el grupo experimental presentó una disminución crónica significativa en la realización de conductas no deseadas en el aula (Beirute, 1974). No habla del efecto de retención.

De igual forma, Mora (1982), comparó un tratamiento de modificación de conducta utilizando el refuerzo positivo con un tratamiento de terapia de juego, para la disminución de la agresividad en niños con daño cerebral, quienes son medicados para disminuir la agresividad. Encontró que ambos tratamientos son efectivos, de forma crónica (pero en mayor medida el refuerzo positivo) y contribuyen a disminuir la dosis de medicamento suministrado para controlar la agresividad. No se refiere al efecto de retención.

En 1998, Silliman y otros trabajaron con siete adultos con retardo mental profundo, utilizando la música como un reforzador positivo, el tono aversivo como un reforzador negativo, y el no refuerzo, en la mejora postural del tren superior del cuerpo. Cinco de los siete participantes prefirieron la música como reforzador, encontrando mayores mejoras en la postura cuando trabajaban con esta condición. Solo dos lograron mejores resultados con el reforzador negativo.

Así mismo, Long y otros (1999), aplicaron un tratamiento simplificado de reversión de hábitos, con personas con retardo mental, quienes disminuyeron hábitos de comerse las uñas y otras conductas oral-digitales.

Sin embargo, queda la inquietud de porqué música-danza no fue eficaz crónicamente, pese a los beneficios encontrados de forma aguda; además de que existe alguna evidencia científica del trabajo de música y/o danza en cuanto a la modificación de conducta con población especial, con resultados favorables tal como lo han hecho Adler (1970), Berrol (1981), Boettinger (1978), Chaiklin (1972), Crain y otros (1984), Silliman (1992). Sin embargo, a pesar de que el tamaño del efecto respalda la superioridad del refuerzo positivo, también se observa un tamaño del efecto alto para música-danza, lo que apoya la inquietud señalada previamente.

No obstante, los alcances logrados con música-danza en el seguimiento de órdenes (otro aspecto conductual), tanto aguda como crónicamente, dan una base alentadora para continuar con su estudio, ya que el aumento en la frecuencia de órdenes deseadas fue muy importante, a pesar del tamaño de la muestra y la variabilidad entre los sujetos. Esto se puede apoyar en los estudios mencionados previamente, donde se trabaja con música y/o danza para mejorar diversos aspectos de la conducta.

De igual forma, el tamaño del efecto confirma los resultados, pero también se observan valores altos en el refuerzo positivo.

En este estudio en particular se debe tomar en cuanta el estado "normal" en que se encuentran los participantes, ya que se mantienen todo el día sin "nada que hacer", por lo tanto, lo único que les queda es la autoestimulación, así que cuando se les ofrecen otras posibilidades, donde aprovechar su tiempo en algo "más productivo o entretenido", es lógico que presten atención a esta nueva opción y dejen un tanto de lado las conductas no deseadas.

Las mejoras crónicas se pueden atribuir, en parte, al hecho de que se dio un tratamiento intensivo, cuatro veces por semana. No hubo efecto de retención, quizás por la corta duración del tratamiento o a la frecuencia y la intensidad utilizadas. No obstante, se ha encontrado en población especial, que la ganancia obtenida en diferentes aspectos medidos (de aptitud física y motriz), se pierde entre los quince días y los tres meses después de haber concluido el tratamiento (Mora y Salazar, 1997; Silliman y otros, 1992).

Los resultados obtenidos son claros, e indican que la música-danza es superior que el refuerzo positivo en tres de las cuatro 
variables analizadas, no obstante hace falta más investigación con diferentes usos de música-danza ya que esto brinda una buena base para considerar que es un método con muchas posibilidades a corto y largo plazo.

\section{Recomendaciones}

Posteriores estudios deben investigar el efecto crónico y la retención utilizando una combinación metodológica de ambos métodos, música-danza, con refuerzo positivo, a la vez que se debe ampliar el tamaño de la muestra.

Se deben investigar los efectos de la música-danza en otras variables conductuales, tales como: comportamiento no verbal, movimientos voluntarios, comunicación (verbal y no verbal) en una población con discapacidad múltiple u otro tipo de discapacidad.

\section{REFERENCIAS}

Adler, J. (1970). The Study of Autistic Children. Proceedings of the Third Annual Conference of the American Dance Therapy Association. 43-48. Alvin, J. (1990). Músico Terapia. $\quad\left(2^{\mathrm{da}}\right.$ edición). Barcelona: Paidós Educador. American Dance Therapy Association (1999). http://www.adta.org.

Beirute, L. (1974). Aplicación de Técnicas de Modificación de Conducta en el Aula, utilizando Maestros como Agentes Terapéuticos. Un Programa de Entrenamiento de no Profesionales en el Campo de la Salud Mental. Tesis no publicada. Escuela de Psicología, Universidad de Costa Rica.

Berrol, F. (1978). The Effects of two Movements Remediation Program on Selected Measures of Perceptual-Motor Ability. Academic Achievement, and Behavior on First Grade Children.

Berrol, F. (1981). A Neurophysiologic Approach to Dance/Movement Therapy: Theory and Practice. American Journal of Dance Therapy. 4 (1), 72-84.

Blockham y Silberman, (1973). Como Modificar la Conducta Infantil. Kapeluz.

Boettinger, J.A. (1978). The Study of the Autistic Child. In M.N. Costonis (De.), Therapy in Motion. Urbana: University of Illinois Press.

Broden, M., Vance, H., Dunlop, A. \& Clark, R. (1970). Efectos de la Atención del Profesor y de un Sistema de Reforzamiento con Fichas en una Clase de
Enseñanza Especial de una Escuela Secundaria. Exceptional Children. 36, 341-349.

Brooks, N. (1989). Children with Learning Disabilities and the Dance/Movement Class. The Journal of Physical Education, Recreation and Dance. 60(9), 59-61.

Bukley, N. y Walker, H. (1970). Modificación de Conducta en el Salón de Clase. Un Manual de Procedimiento para el Maestro. Instituto Interamericano de Estudios Psicológicos y Sociales. México.

Cabanas, R. (1979). A cerca de una Teoría sobre el Origen del Habla en la Humanidad con Derivaciones Terapéuticas: Nueva Interpretación. La Habana: Ministerio de Salud Pública. Hospital Psiquiátrico de la Habana.

Cabezas, H. y Fonseca, G. (1992).

Entrenamiento en la Adquisición de la Imitación Verbal en Tres Niños Autistas. Revista Educación. 16(1), 101-106.

Cabezas, H. (1989). El uso de Técnicas de Modificación de Conducta para la Adquisición de Destrezas Básicas y Pre-académicas. Tesis no publicada. Escuela de Educación, Universidad de Costa Rica.

Calder, J. (1972). Dance for Mentally Retarded. Slow Learning Child. 19(2), 67-78.

Carvajal, T. (1989). Un Programa de Entrenamiento Teórico-Práctico para Maestros de Escuelas Primarias Públicas, en Técnicas Básicas de Modificación de Conducta en el Aula. Tesis no publicada. Escuela de Psicología. Universidad de Costa Rica.

Chaiklin, H. (1974). Multiple Theoretical Perspectives in Interpreting Behavior. Proceedings of the Ninth Annual Conference of the American Dance Therapy Association. 48-58.

Crain, C., Eisenhart, M. \& Mc Laughlin, J. (1984). The Application of a Multiple Measurement Approach to Investigate the Effects of a Dance Program on Educable Mentally Retarded Adolescents. Research Quarterly for Exercise and Sport. 55(3), 231236.

Culver, A. (1989). Comparison of Behavior Modification Techniques Used in Physical Education with Institutionalized Profoundly Mentally Retarded Students at Different Ages. Unpublished Master's Thesis, Texas Woman's University, Denton.

Díaz, P. (editor) (1994). Memorias del Seminario Konrad Lorenz sobre Etología. Santiago, Santa Fe de Bogotá: Academia Colombiana de Ciencias Exactas, Física y Naturales.

Dorney, 1., Gho, E. K. \& Lee, C. (1992). The Impact of Music and Imagery on Physical Performance and Arousal: Study of Coordination and Endurance. Journal of Sport Behavior. 15(1), 21-33.

Eibi-Eibesfeldt, I. (1974). Etología. Introducción al Estudio Comparado del Comportamiento. ( $1^{\text {ra }}$ edición en español). Barcelona: Omega. 
Franks, C. Caballo, E. (compilador), (1991). Manual de Técnicas de Terapia y Modificación de Conducta. Madrid: Siglo XXI de España Editores. España.

Ferster, C. (1961). Positive Reinforcement and Behavioral Deficits of Autistic Children. Child Development. 32, 437-456.

Freundlich, B., Pike, L. \& Schwartz, V. (1989). Dance and Music for Children with Autism. The Journal of Physical Education, Recreation and Dance. 60 (9), 50-53.

Frieder, S. and Ekstein, D. (1981). What is

Dance Therapy? Anual for Facilitators, and Consultantes. American Dance Therapy Association. Granell, E. (1979). Aplicación de Técnicas de Modificación de Conducta: para el Control de la Hiperactividad en el Ambiente Natural. México, D.F: Trillas. México.

Hall, E. (1989). La Dimensión Oculta. (13 edición). México. D.F: Siglo Veintiuno.

Jatib, W., Bustillo, M. \& Jinete, M. (1998). Incidencia de la Música en el Desarrollo de la Atención, la Memoria y la Percepción del Niño con Retraso Mental Integrado al Aula Regular. Artículo sin Publicar. Simposio de Problemas de Aprendizaje. Bogotá.

Jay, D. (1991). Effect of a Dance Program on the Creativity of Preschool Handicapped Children. Adapted Physical Activity Quarterly. 8(4), 305-316.

Kavaler, S. (1974). The Effects of Dance Therapy on Mentally Retarded Children. (Doctoral Dissertation, Adelphi University). Dissertation Abstract International, 35, 2435-B

Keppel, G. (1982). Design and Analysis a Researcher's Handbook. New Jersey: Prentice-Hall. Kraus, R. (1978). The Therapeutic Recreation Service: Principles and Practice. Philadelphia: W. B. Saunders Company.

Levy, F. (1988). Dance Movement Therapy: A Healing Art. Reston, VA: The American Alliance for Health, Physical Education, Recreation and Dance.

Long, E. S., Miltenberger, R. G. \& Ellingson, S. A. (1999). Augmenting Simplified Habit Reversal in the Treatment of Oral-Digital Habits Exhibited by Individuals with Mental Retardation. Journal of Applied Behavior Analysis. 32(3), 353-365.

Lorenz, K. (1984). Los Ocho Pecados Mortales de la Humanidad Civilizada. ( ${ }^{\text {ra }}$ edición). Barcelona: Plaza \& Jones.

Loroño, A. y del Campo, P. (1987). Taller de Biomúsica. Manual de Músico terapia. Cuaderno nº de la Revista Integral. ( $1^{\text {ra }}$ edición). Barcelona.

Masako, O. and Chiyo, K. (1996). Influence of the Dance Stimulus on Response to a Music Piece. Bulletin of Institute of Health and Sport Science, University of Tsukuba. 19, 75-84.

Mora, A. (1982). La Terapia de Juego y la Modificación de Conducta como Coadyuvantes al Tratamiento Medicamentoso de la Agresividad en Niños con Daño Cerebral en el Hospital México. Tesis no publicada. Escuela de Psicología, Universidad de Costa Rica.

Mora, D y Salazar, W, (1997). Efectos del Entrenamiento y Desentrenamiento en la Retención de Destrezas y Habilidades en Sujetos con Retraso en el Desarrollo Psicomotor. Artículo sin Publicar. Memoria del IV Simposio Internacional en Ciencias del Deporte y la Salud, Escuela de Educación Física y Deportes, Universidad de Costa Rica.

Mora, D. y Salazar, W. (1999). El Efecto de la Música y la Danza en la Conducta de Personas con Discapacidad Múltiple Severa. Kinesis: Revista de Rehabilitación Integral. 3, 23-29.

Moran, J. and Kalakian, L. (1974). Movement Experiences for the Mentally Retarded on Emotionally Disturbed Child. Minneapolis, MN: Burgues Publishing Company.

Neddo, J.M. (1986). Effects of Exercises on Type A Behavior Pattern. Microform Publications, University of Oregon, Eugene, Ore.

Ohwaki, S. (1976). An Assessment of Dance Therapy to Improve Retarded Adults`Body Image. Perceptual and Motor Skills. 43, 1122.

Ozols, M.A. y Revuelta, I. (1996). La Danza y la Natación como una Herramienta en el Proceso de Aprendizaje del Niño y el Joven con Discapacidad. Artículo sin Publicar. V Simposium Internacional en Ciencias del Deporte y la Salud. Universidad de Costa Rica.

Paniagua, E. (1994). Efectos de las técnicas de modificación de conducta en la disciplina de los alumnos de segundo grado del Instituto de Psicopedagogía Integra. Tésis no publicada. San José: Escuela de Formación Docente, Facultad de Educación, Universidad de Costa Rica.

Piaget, J. (1989). Adaptación Vital y Psicología de la Inteligencia: Selección Orgánica y Fenocopia. $\quad\left(7^{\mathrm{ma}}\right.$ edición). México. D.F: Siglo Veintiuno.

Piaget, J (1994). Biología y Conocimiento: Ensayo. ( $2^{\mathrm{ma}}$ edición). México. D.F: Siglo Veintiuno.

Piazza, C.C., Adelinis, J.D. \& Hanley, G.P. (2000). An Evaluation of the Effects of Matches Stimuli on Behaviors Maintained by Automatic Reinforcement. Journal of Applied Behavior Analysis. 33(1), 13-27.

Ribes, E. (1975). Técnicas de Modificación de Conducta, su Aplicación al Retardo del Desarrollo. México. D.F; Trillas.

Ricci, P. y Cortesi, S. (1979). Comportamiento no Verbal y Comunicación. Barcelona: Gustavo Gili, S.A.

Riordan, A. (1989). Sunrise Wheels. The Journal of Physical Education, Recreation and Dance. $60(9), 62-64$.

Schmais, C. (1974). Dance Therapy in Perspective. In focus on Dance. American Association for Health, Physical, Education and Recreation. VII, 712. 
Siedentop, D. and Rushall, B. (1972). The Development and Control of Behavior in Sport and Physical Education. Philadelphia: Lea \& Febiger.

Silliman, L., French, R. \& Tynan, D. (1992).

Use of Sensory Reinforcement to Increase Compliant Behavior of a Child Who is Blind and Profoundly Mentally Retarded. Clinical Kinesiology. 46(3), 3-9.

Silliman, M., French, R., Sharrill, C. \& Gench, B. (1998) Auditory Feedback and Time-onTask of Postural Alignment of Individuals with Profound Mental Retardation. Adapted Physical Activity Quarterly. 15(1), 51-63.

Skinner, B.(1975). Sobre el Conductismo.

Barcelona: Fontanella, S.A.

Stranathan, P., French, R. \& Kinnison, L. (1997). Behavior Modification Techniques used and preferred to be used in Physical Education in the Public School with Students who are Profoundly Mentally Retarded. Paper presenter at the $5^{\text {th }}$ National Conference on adapted Physical Activity. Macomb, IL. Sulzer-Azaroff, B. y Mayer, R. (1988). Procedimientos del Análisis Conductual Aplicado con Niños y Jóvenes. México: Trillas S.A.

Terán, D. (1998). Arte, Música y Músicoterapia. Minusval. 19(81), 35-37.

Thomas, J., Salazar, W. \& Landers, D. (1991). What is Missing in p<.05? Effect Size. Research Quarterly for Exercise and Sport. 62(3), 344348.

Walker, J. y Shea, T. (1987). Manejo Conductual. México D.F: El Manual Moderno, S.A. México.

Weltman, M. (1986). Movement Therapy with Children who have been Sesually Abused. American
Journal of Dance Therapy, 9, 47-66.Ellis, D. and Cress, P. (1993). Training Students with Mental Retardation to Self-Pace While Exercising. Adapted Physical Activity Quarterly. 10, 104-124.

Wise, S. K. (1981). Integrating the use of Music in Movement Therapy for Patients with Spinal Cord Injuries. American Journal of Dance Therapy, 4 (1), 42-51. 